\title{
Avaliação dos critérios de projeto da Norma Brasileira NBR 12209/2011 aplicados a filtros biológicos percoladores com meio suporte plástico \\ Assessment of the Brazilian Standard NBR 12209/2011 design criteria of Trickling Filters with plastic packing medium \\ Data de entrada: $10 / 07 / 2018$ \\ Data de aprovação: 04/12/2019
}

Ana Silvia Pereira Santos ${ }^{1 * *} \mid$ Eduardo Fazza Dielle $^{2} \mid$ Renata de Oliveira Pereira $^{2} \mid$ Sue Ellen Costa Bottrel ${ }^{2}$

DOI: https://doi.org/10.36659/dae.2021.010

ORCID ID

Santos ASP (D) https://orcid.org/0000-0001-7823-9837

Dielle EF (D) https://orcid.org/0000-0003-4293-578X
Pereira RO (ID) https://orcid.org/0000-0002-3414-7292 Bottrel SEC (iD) https://orcid.org/0000-0001-9755-4074

\section{Resumo}

Na Norma Brasileira NBR 12.209/2011, o Filtro Biológico Percolador - FBP apresenta a possibilidade de uso de meio suporte plástico, com novos valores para os parâmetros de referência. Para tanto, os parâmetros de projeto, como a carga orgânica volumétrica (COV), a taxa de aplicação superficial (TAS) e a profundidade do filtro $(\mathrm{H})$, foram alterados para se adequarem melhor às particularidades desse meio suporte. Nesse sentido, este trabalho visa apresentar uma análise crítica dos valores limites estabelecidos para esses parâmetros, por meio de cálculos teóricos. Observou-se que as vantagens associadas à utilização do meio suporte plástico, de possibilidade de aplicação de COV elevada e de elevada profundidade de meio suporte (até $12 \mathrm{~m}$ ), com a consequente redução de área ocupada, não são facilmente alcançadas. Ao se elevar a profundidade do meio suporte ao limite determinado pela Norma, alcança-se uma carga orgânica volumétrica bastante inferior àquela sugerida para este fim e taxas de aplicação superficial superiores ao limite máximo recomendado. Alcança-se no máximo 9,0 m de profundidade, considerando-se o FBP precedido pelo Reator UASB, com TAS $=75 \mathrm{~m}^{3} \cdot \mathrm{m}^{-2} \cdot \mathrm{d}^{-1}$ e COV = 1,0 kgDBO $\cdot \mathrm{m}^{-3} \cdot \mathrm{d}^{-1} \cdot$ Para a mesma TAS e COV = 1,5 kgDBO $\cdot \mathrm{m}^{-3} \cdot \mathrm{d}^{-1}$ foi possível alcançar somente 6,0 $\mathrm{m}$ de profundidade de meio suporte.

Palavras-chave: Tratamento de esgoto. Carga orgânica volumétrica. Taxa de aplicação superficial. Profundidade do filtro.

\section{Abstract}

In Brazilian Standard NBR 12.209/2011, trickling filters (TF) present the possibility of using plastic-based packing media, with new values for the reference parameters. Therefore, the design parameters, such as organic loading rate $(O L R)$, hydraulic loading rate $(H L R)$ and filter depth $(H)$, were altered to better suit the particularities of this support medium. In this sense, this work aims to present a critical analysis of the limit values established for these parameters, through theoretical calculations. It was observed that the use of plastic-based packing media allows higher $\mathrm{H}$ (up to $12 \mathrm{~m}$ ) and lower footprint. However, the possibility of applying higher OLR (main advantage) is not

\footnotetext{
${ }^{1}$ Universidade do Estado do Rio de Janeiro - Rio de Janeiro - Rio de Janeiro - Brasil.

${ }^{2}$ Universidade Federal de Juiz de Fora - Juiz de Fora - Minas Gerais - Brasil.

* Autora correspondente: anasilvia.lightneragmail.com.
} 
easily achieved. When the $H$ is raised to the maximum value recommended in the standard, OLR is far from the minimum referenced for TF, resulting in $H L R$ highest than recommended in the standard. Considering TF preceded by UASB reactors, $H=9 \mathrm{~m}$ is the highest that leads to $H L R=75 \mathrm{~m}^{3} \cdot \mathrm{m}^{-2} \cdot \mathrm{d}^{-1}$ and $O L R=1,0 \mathrm{kgDBO} \cdot \mathrm{m}^{3} \cdot \mathrm{d}^{-1}$ as recommended in the standard. Using the same HLR and OLR $=1,5 \mathrm{kgDBO} \cdot \mathrm{m}^{-3} \cdot \mathrm{d}^{-1}$, it is reached a H of only $6 \mathrm{~m}$.

Keywords: Wastewater treatment. Organic loading rate. Hydraulic loading rate. Filter depth.

\section{INTRODUÇÃO}

A importância do tratamento de esgotos associado às condições adequadas de saneamento é indispensável para a manutenção da qualidade ambiental e a preservação da saúde humana. 0 lançamento de efluentes em corpos d'água sem tratamento prévio contribui para a degradação dos mesmos e para a proliferação de diversos tipos de doenças parasitárias e infecciosas.

Para que os projetos das Estações de Tratamento de Esgoto - ETE sejam elaborados de forma eficiente, é indispensável que o responsável tenha capacitação técnica e recursos suficientes. No entanto, segundo Jordão e Pessoa (2017), as novas estações de tratamento não se restringem apenas às exigências ambientais, de saúde pública, estéticas ou legais. Nesses casos são também consideradas de forma equitativa exigências tecnológicas, econômicas e mesmo os anseios da comunidade. Dessa forma, os responsáveis pelos projetos devem manter cada vez mais uma sintonia entre amplo conhecimento tanto das informações disponíveis na bibliografia como do instrumento que norteia os projetos de ETE no Brasil. Nesse contexto, encontra-se a Norma Brasileira NBR 12.209/2011, editada pela Associação Brasileira de Normas Técnicas (ABNT), que estabelece parâmetros e apresenta as condições recomendadas para a elaboração de projeto hidráulico e sanitário de processos de Estações de Tratamento de Esgotos.

A NBR 12.209/2011 representa um avanço na área de tratamento de esgotos domésticos, principalmente se comparada à Norma anterior, de 1992 (ABNT, 1992). A nova Norma contempla tanto tecnologias mais consagradas no país (lodos ativados e filtros biológicos percoladores) como outras ainda menos difundidas (tecnologias de filtros aerados submersos e biofiltros aerados submersos), além da tecnologia híbrida conhecida como Reator Biológico de Leito Móvel. Além disso, esta contempla o reator UASB - Upflow Anaerobic Sludge Blanket, atualmente bastante utilizado em projetos de ETE no Brasil (ANA, 2017).

Dessa forma, a nova NBR 12.209/2011 vem sendo cada vez mais difundida. Entretanto, muitos projetos são realizados apenas de forma a respeitar os padrões da Norma e obter licenças ambientais, principalmente em pequenos municípios que possuem dificuldades de acesso à mão-de-obra técnica qualificada. Além da adequação aos parâmetros pré-estabelecidos pela NBR 12.209/2011, a escolha das tecnologias pelos projetistas deve passar por um estudo que considera tanto as características dos processos como as particularidades do local onde este será executado, de forma a torná-lo mais eficiente.

Alguns estudos recentes visam auxiliar no processo de tomada de decisão por parte de gestores de saneamento. Nesse sentido, pesquisadores da Universidade de São Paulo (USP) desenvolveram um software denominado ETEX®, que tem o objetivo de auxiliar gestores municipais na escoIha dos processos mais adequados em função de custos de implantação, operação e manutenção, por meio da comparação entre 6 dos principais fluxogramas de tratamento de esgotos adotados no Brasil (USP, 2018). Ainda nesse contexto, Borges (2017) estimou as quantidades dos principais 
serviços e equipamentos necessários para a construção de 6 diferentes fluxogramas de ETEs que atendam técnica e economicamente a municípios brasileiros de pequeno porte (faixas populacionais de 5 mil a 50 mil habitantes). 0 referido estudo possibilita a adoção de preços unitários para serviços e equipamentos para, por fim, estimar o valor global de implantação da unidade completa.

Uma das tecnologias de tratamento de esgotos abordadas pela NBR $12.209 / 2011$, os Filtros Biológicos Percoladores - FBPs, destinados à remoção de matéria orgânica, estão presentes em muitas estações de tratamento em operação no Brasil. Segundo ANA (2017), 424 das 2657 ETEs em operação no Brasil em 2013 adotavam os filtros aeróbios em seus fluxogramas. Destas, 185 adotavam o fluxograma composto por reator UASB seguido de filtros aeróbios, dentre eles FBP. Na Região Metropolitana de Belo Horizonte, no estado de Minas Gerais, encontra-se em operação desde 2006 a ETE Onça, projetada para tratar o esgoto de 2,0 milhões de habitantes, com vazão de projeto de $1,8 \mathrm{~m}^{3} \cdot \mathrm{s}^{-1}$, com o fluxograma composto por UASB-FBP (COPASA, 2006). Segundo Vieira (2013), esta é uma das maiores estações de tratamento de esgotos do mundo, e a maior do país com essa tecnologia, composta por 24 reatores UASB e 8 FBPs, além dos decantadores secundários. De acordo com pesquisa realizada por Moraes et al. (2011), na ETE Onça, os FBPs são preenchidos com pedra britada, em altura útil de 2,5 m e no período de dezembro de 2009 a março de 2011 apresentaram eficiências globais medianas de $85 \%$, $85 \%$ e $86 \%$ de remoção de Demanda Química de Oxigênio (DQO), Demanda Bioquímica de Oxigênio (DBO) e Sólidos em Suspensão Totais (SST), respectivamente.

Os FBPs, conforme apresentado na Fig. 1, são constituídos de grandes tanques preenchidos com material inerte, denominado "meio suporte", onde a biomassa responsável pela degradação da matéria orgânica presente nos esgotos tem facilidade em aderir e formar um filme biológico conhecido como biofilme (JORDÃO E PESSÔA, 2017). A aplicação dos esgotos em unidades circulares deve ser uniforme sobre a superfície do meio suporte por meio do distribuidor rotativo, garantindo a melhor taxa de molhamento possível (ABNT, 2011; SANTOS, 2005). Após distribuição, o esgoto percola continuamente pelos interstícios do meio suporte e, dessa forma, favorece o crescimento e a aderência de microrganismos. Por meio do contato do esgoto afluente com a massa biológica do meio suporte, os microrganismos retêm a matéria orgânica pelo fenômeno de adsorção, degradando-a por meio de uma oxidação bioquímica. A remoção da matéria orgânica ocorre pela transformação das substâncias dissolvidas e coloidais, presentes no esgoto, em flocos de sólidos com boa sedimentabilidade, que serão removidos posteriormente nos tanques de decantação secundária (JORDÃO E PESSÔA, 2017).

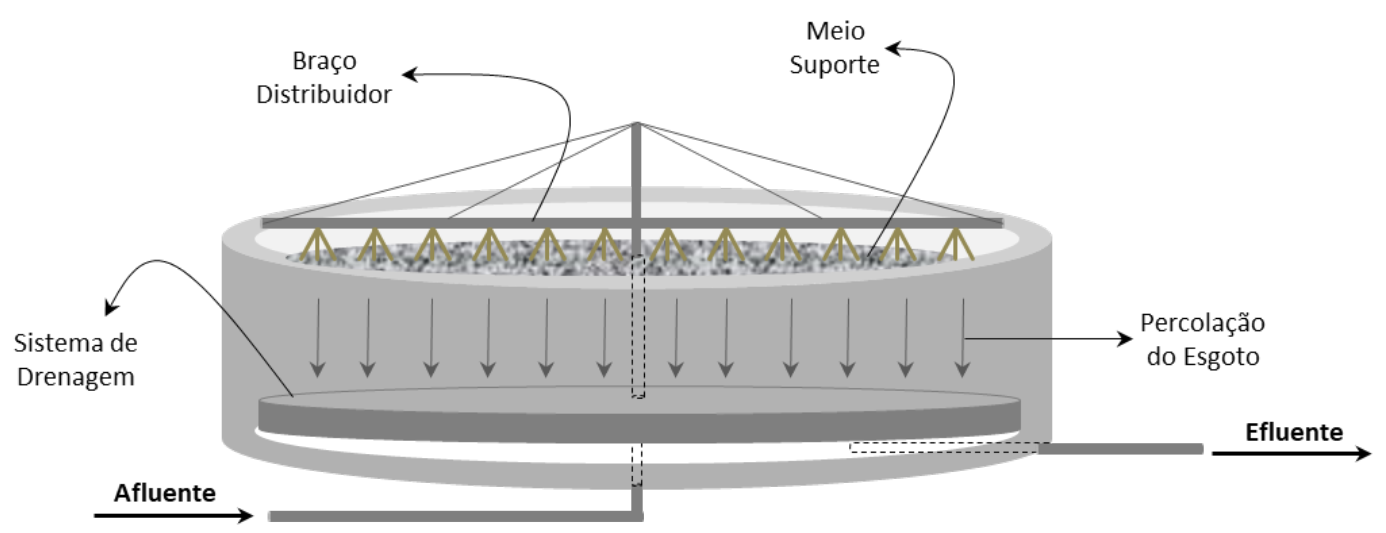

Figura 1 - Desenho esquemático do Filtro Biológico Percolador Fonte: os autores 
De acordo com a NBR $12.209 / 2011$, os FBPs podem ser dimensionados para receber menor (no máximo 0,3 $\mathrm{kgDBO} \cdot \mathrm{m}^{-3} \cdot \mathrm{d}^{-1}$ - meio suporte em pedra britada) ou maior (no máximo 3,0 $\mathrm{kgDBO} \cdot \mathrm{m}^{-3} \cdot \mathrm{d}^{-1}$ - meio suporte em plástico) quantidade de matéria orgânica por unidade de volume, caracterizando uma unidade de baixa ou alta carga, respectivamente. Nos casos em que a carga orgânica aplicada é baixa, a respiração endógena tende a acontecer, podendo gerar um lodo parcialmente estabilizado. Nessas condições há uma redução considerável de matéria orgânica carbonácea, o que viabiliza a atuação dos organismos nitrificantes que oxidam o nitrogênio amoniacal a nitrito e nitrato, podendo ocorrer a nitrificação (SPERLING, 2016). Em concordância com o exposto por Sperling (2016), Almeida (2007) analisa as eficiências de remoção de FBP preenchidos com pedra (COV de 0,2 a $\left.0,8 \mathrm{kgDBO} \cdot \mathrm{m}^{-3} \cdot \mathrm{d}^{-1}\right)$, chegando a efluentes parcialmente nitrificados, com remoção de até $50 \%$ de nitrogênio amoniacal na menor COV. Como a comunidade microbiológica recebe menor quantidade de matéria orgânica em uma unidade de baixa carga, o desempenho desta é levemente superior ao desempenho da unidade de alta carga. Entretanto, essa aplicação de uma carga mais baixa por unidade de área leva a unidades que ocupam áreas mais elevadas. De maneira oposta, no caso de filtros de alta carga, os requisitos de área são inferiores, já que se permite maior carga hidráulica por unidade de área. Ainda, é possível observar uma ligeira redução na eficiência de remoção de matéria orgânica e a formação de um lodo não estabilizado. Por fim, nos filtros de alta carga, é necessária a recirculação do efluente para manter vazão uniforme, de forma a equilibrar a carga afluente e possibilitar nova chance de contato entre a matéria orgânica remanescente e o biofilme (JORDÃO E PESSOA, 2017; SANTOS, 2005; VIEIRA, 2013; VIEIRA E SPERLING; 2012). Segundo Duda e Oliveira (2011), nos experimentos conduzidos em filtros biológicos de alta taxa com recirculação de $200 \%$, foram obtidas as maiores remoções de DQO total. Neste caso, os autores afirmam que a recirculação foi necessária para melhorar a estabilidade do sistema e manter as eficiências de remoção de DQ O total, SST e SSV acima de 93\%.

Ressalta-se que o meio suporte normalmente utilizado nos filtros sempre foi composto por pedra britada número 4. A partir dos anos 2000, vem sendo indicado por muitos pesquisadores (SANTOS, 2005; CHERNICHARO et al., 2006; RIBEIRO et al., 2018) e fabricantes a utilização do meio suporte plástico, que incorpora diversas vantagens em relação à pedra, porém apresenta um custo mais elevado e ainda encontra dificuldades para ser absorvido pelos prestadores de serviço de água e esgoto no Brasil (SANTOS, 2005).

Segundo Henrich e Maeggraff (2013), no final da década de 70, diferentes configurações de meio suporte plástico para filtros percoladores já eram estudadas. A evolução dessas experiências resultou em novas gerações de FBP que, utilizando meio suporte plástico em substituição à pedra brita, podem superar problemas relacionados à colmatação do filtro e ao mesmo tempo proporcionar maior capacidade de remoção de matéria orgânica.

Ainda, o meio suporte plástico admite, com menor área superficial, a aplicação de cargas orgânicas mais elevadas, resultando ainda na possibilidade de se alcançar unidades mais esbeltas. Comparado à pedra, o meio suporte plástico apresenta maior índice de vazios, maior área superficial específica e menor peso específico. Essas características permitem unidades de filtração biológica com profundidades mais elevadas, garantindo, mesmo assim, o livre fluxo de oxigênio no interior do tanque (SANTOS, 2005).

Os principais parâmetros de dimensionamento do FBP são a Carga Orgânica Volumétrica - COV e a Taxa de Aplicação Superficial - TAS. A COV re- 
laciona a massa de matéria orgânica presente no esgoto afluente ao filtro, durante um dia $\left(\mathrm{kg}^{\mathrm{d}} \mathrm{d}^{-1}\right)$, com o volume útil do tanque $\left(\mathrm{m}^{3}\right)$. Já a TAS representa a vazão $\left(\mathrm{m}^{3} \cdot \mathrm{d}^{-1}\right)$ por unidade de área superficial do tanque $\left(\mathrm{m}^{2}\right)$.

Assim, devem-se adotar, para dimensionamento do FBP, valores de COV e TAS diferentes quando se trata de utilização de meio suporte plástico ou em pedra, ou ainda em casos de alta ou baixa carga. Porém, essas indicações para o caso de utilização de meio plástico somente foram incorporadas na última revisão da NBR 12.209 de 2011. A versão anterior da NBR até mencionava a possibilidade de utilização de outros materiais, porém indicava que os parâmetros e critérios adotados deveriam ser justificados.

Na Tabela 1, podem-se observar os parâmetros de dimensionamento (COV, TAS e Profundidade Útil) indicados pela NBR 12.209 tanto para a versão de 1992 como para a última revisão de 2011, em se tratando de filtros com meio suporte plástico ou em pedra, e alta ou baixa carga. Ressaltase que na edição de 2011 a NBR 12.209 indica o meio suporte plástico somente para filtros de alta carga, garantindo o principal benefício associado a esse tipo de meio, que é a possibilidade de aplicação de cargas mais elevadas.

Tabela 1 - Resumo dos parâmetros recomendados pelas NBR 12.209/1992 e NBR 12.209/2011 para dimensionamento do FBP em função do meio suporte

\begin{tabular}{|c|c|c|c|c|}
\hline NBR & Material Suporte & $\begin{array}{c}\text { TAS } \\
\left(m^{3} \cdot m^{-2} \cdot d^{-1}\right)\end{array}$ & $\begin{array}{c}\text { COV } \\
\left(\mathrm{kgDBO} \cdot \mathrm{m}^{-3} \cdot \mathrm{d}^{-1}\right)\end{array}$ & $\begin{array}{l}\text { Profundidade } \\
\text { (m) }\end{array}$ \\
\hline \multirow{2}{*}{$\begin{array}{l}12.209 \\
(1992)\end{array}$} & Pedra britada ou seixo rolado & $\begin{array}{c}0,8-5,0(B C) \\
10,0-60,0(A C)\end{array}$ & $\begin{array}{l}<0,3(B C) \\
<1,8(A C)\end{array}$ & $<6,0$ \\
\hline & Outros materiais & \multicolumn{2}{|c|}{ Os parâmetros e critérios devem ser justificados } & $<6,0$ \\
\hline \multirow{2}{*}{$\begin{array}{l}12.209 \\
(2011)\end{array}$} & Pedra britada & $\begin{array}{l}<5,0(B C) \\
<50,0(A C)\end{array}$ & $\begin{array}{l}<0,3(B C) \\
<1,2(A C)\end{array}$ & $<3,0$ \\
\hline & Meio plástico & $10,0-75,0$ & $<3,0$ & $<12,0$ \\
\hline
\end{tabular}

Legenda: BC - Baixa Carga; AC - Alta Carga; COV - Carga Orgânica Volumétrica; TAS - Taxa de Aplicação Superficial.

Tanto a COV como a TAS são parâmetros imprescindíveis na avaliação do desempenho dos FBP. Valores baixos de COV e TAS podem resultar em um fornecimento insuficiente de substrato para os microrganismos, levando à morte dos mesmos. Todavia, valores muito elevados podem sobrecarregar o sistema, fazendo com que não haja capacidade suficiente de tratamento e, consequentemente, um baixo desempenho (JORDÃO E PESSOA, 2017).

Por fim, essas unidades normalmente são dimensionadas a partir da adoção de um valor para COV ou TAS, recomendados pela Norma, devendo-se fixar também um valor para a altura do meio suporte $(\mathrm{H})$. Caso o dimensionamento seja realizado pela TAS, deve-se verificar se a COV encontra-se dentro da faixa recomendada pela NBR 12.209/2011. E caso o dimensionamento seja efetuado pela COV, a TAS deve ser verificada.

Dessa forma, o principal objetivo do presente trabalho foi analisar os limites máximos e mínimos para os parâmetros de dimensionamento do filtro biológico percolador (TAS, COV e H), indicados na mais recente revisão da NBR 12.209 de 2011 para meio suporte plástico. Essa análise se deu em função da necessidade de avaliação da aplicação dessas taxas para alcançar os benefícios relacionados à adoção do meio suporte plástico, como aplicação de maior carga orgânica volumétrica, possibilidade de ocupação de menores áreas e adoção de profundidades de meio suporte mais elevadas. 


\section{METODOLOGIA}

Com o intuito de verificar as recomendações da NBR 12.209 (2011) para o FBP de alta taxa com meio suporte plástico, foram analisados os limites inferiores e superiores dos parâmetros "COV", "TAS" e "H" de forma a diagnosticar as interferências de cada um sobre o outro no dimensionamento dos filtros, apresentando valores condizentes com as recomendações da própria Norma para os parâmetros mencionados.

Segundo Jordão e Pessoa (2017), os parâmetros COV e TAS são representados pelas Eq. 1 e 2, respectivamente.

$\operatorname{COV}=\frac{C}{V}$

Onde:

COV - Carga Orgânica Volumétrica $\left(\mathrm{kgDBO} \cdot \mathrm{m}^{-3} \cdot \mathrm{d}^{-1}\right)$

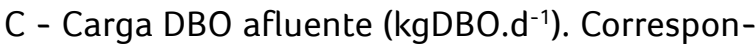
dente à Vazão Afluente $x$ Concentração DBO afluente

$\mathrm{V}$ - Volume $\left(\mathrm{m}^{3}\right)$

$T A S=\frac{Q}{A}$

Onde:

TAS - Taxa Aplicação Superficial $\left(\mathrm{m}^{3} \cdot \mathrm{m}^{-2} \cdot \mathrm{d}^{-1}\right)$

Q - Vazão $\left(\mathrm{m}^{3} \cdot \mathrm{d}^{-1}\right)$

A - Área $\left(\mathrm{m}^{2}\right)$

Assim, as Eq. 1 e 2 foram agrupadas em uma única equação (Eq. 3) para o dimensionamento do FBP com as variáveis correspondentes aos 3 parâmetros de dimensionamento já mencionados, variando em função da concentração afluente de DBO.

$C O V=\frac{D B O \times T A S}{H}$
Onde:

COV - Carga Orgânica Volumétrica $\left(\mathrm{kgDBO} \cdot \mathrm{m}^{-3} \cdot \mathrm{d}^{-1}\right)$ DBO - Concentração afluente de DBO $\left(\mathrm{kgDBO}^{-3} \mathrm{~m}^{-3}\right)$ TAS - Taxa Aplicação Superficial $\left(\mathrm{m}^{3} \cdot \mathrm{m}^{-2} \cdot \mathrm{d}^{-1}\right)$ $\mathrm{H}$ - Altura do meio suporte (m)

A partir da Eq. 3, foram definidas seis condições operacionais, realizadas por cálculos teóricos, onde se buscou avaliar, com a aplicação de cargas orgânicas mais elevadas, a possibilidade de alcançar reduzida ocupação de área, considerando-se que a utilização do meio plástico permite adoção de maiores profundidades. Ressalta-se que, neste caso, conforme apresentado na Tabela 1, a NBR 12.209/2011 indica a possibilidade de adoção de profundidades bastante elevadas, de até $12 \mathrm{~m}$ de altura.

Em cada uma das seis condições operacionais teóricas, foram fixados valores para COV e concentração afluente de DBO. No caso da COV, foram adotados valores entre 0,5 $\mathrm{kgDBO} \cdot \mathrm{m}^{-3} \cdot \mathrm{d}^{-1}$ e 3,0 $\mathrm{kgDBO} \cdot \mathrm{m}^{-3} \cdot \mathrm{d}^{-1}$, sendo este último o valor máximo recomendado pela Norma. Para a concentração afluente de DBO à unidade de filtração, foram adotados valores de $120 \mathrm{mg} \cdot \mathrm{L}^{-1}$ ou $210 \mathrm{mg} \cdot \mathrm{L}^{-1}$. Esses valores variam em função da unidade primária adotada no fluxograma completo e partindo-se de uma concentração média afluente à ETE de $300 \mathrm{mgDBO} \cdot \mathrm{L}^{-1}$. Esse é um valor típico em esgotos domésticos, segundo Sperling (2005). Assim, a utilização do reator UASB como pré-tratamento sugere uma eficiência de remoção de matéria orgânica em torno de $60 \%$ considerando apenas essa unidade (JORDÃO E PESSOA, 2017) e consequentemente um afluente ao filtro com DBO de $120 \mathrm{mg} \cdot \mathrm{L}^{-1}$. No caso da adoção do fluxograma convencional com decantador primário (DP), sugere-se uma eficiência em torno de $30 \%$ (JORDÃO E PESSOA, 2017), apresentando como consequência um afluente ao filtro com DBO de $210 \mathrm{mg}^{\mathrm{L}} \mathrm{L}^{-1}$. 
Para cada uma dessas condições operacionais teóricas, adotou-se para efeito de cálculo COV variando entre 0,5 e 3,0 $\mathrm{kgDBO} \cdot \mathrm{m}^{-3} \cdot \mathrm{d}^{-1}$ e DBO de $120 \mathrm{mg} \cdot \mathrm{L}^{-1}$ ou $210 \mathrm{mg} \cdot \mathrm{L}^{-1}$, em função da tecnologia de tratamento precedente ao filtro, conforme explicado anteriormente. Assim, as seis condições operacionais teóricas foram determinadas conforme dados apresentados na Tabela 2. Ressaltase que os valores foram adotados simplesmente para efeito de cálculo teórico, e dessa forma os intervalos estão irregulares, já que se priorizaram principalmente os limites máximos e mínimos. Observa-se, assim, que no caso da adoção do decantador primário como unidade precedente ao FBP, adotaram-se para cálculo teórico somente os valores de 1,0 e 3,0 $\mathrm{kgDBO} \cdot \mathrm{m}^{-3} \cdot \mathrm{d}^{-1}$, já que essa configuração (DP + FBP) encontra-se atualmente em desuso para novos projetos.

Tabela 2 - Principais aspectos relacionados às diferentes condições operacionais teóricas de estudo

\begin{tabular}{|c|c|c|c|}
\hline Condição operacional teórica & $\operatorname{COV}\left(\mathrm{kgDBO} \cdot \mathrm{m}^{-3} \cdot \mathrm{d}^{-1}\right)$ & $\begin{array}{l}\text { Concentração de DBO afluente } \\
\left.\text { (mg. } \mathrm{L}^{-1}\right)\end{array}$ & Unidade de pré-tratamento \\
\hline 1 & 0,5 & 120 & Reator UASB \\
\hline 2 & 1,0 & 120 & Reator UASB \\
\hline 3 & 1,5 & 120 & Reator UASB \\
\hline 4 & 3,0 & 120 & Reator UASB \\
\hline 5 & 1,0 & 210 & Decantador Primário \\
\hline 6 & 3,0 & 210 & Decantador Primário \\
\hline
\end{tabular}

Legenda: COV - Carga Orgânica Volumétrica; DBO - Demanda Bioquímica de Oxigênio.

Por fim, para cada uma das condições operacionais teóricas, foram realizados dois cálculos. No primeiro, adotou-se TAS no valor máximo recomendado pela Norma $\left(75 \mathrm{~m}^{3} \cdot \mathrm{m}^{-2} \cdot \mathrm{d}^{-1}\right)$, com o objetivo de calcular a profundidade para essa situação. E, no segundo, a profundidade $(H)$ foi fixada também no valor máximo recomendado pela Norma (12 m) com o objetivo de calcular TAS nessa outra situação.

\section{RESULTADOS E DISCUSSÕES}

A Tabela 3 apresenta os parâmetros adotados em cada uma das seis condições operacionais teóricas e de maneira destacada (negrito), os resultados para TAS e H em cada uma das situações impostas pela adoção daqueles parâmetros. Ressalta-se que os valores para TAS ou $\mathrm{H}$ tachados, nas duas últimas colunas, encontram-se fora dos limites estabelecidos pela NBR 12.209/2011.

Tabela 3 - Estimativas dos valores de TAS e H em diferentes condições operacionais teóricas para a operação do FBP

\begin{tabular}{|c|c|c|c|c|c|c|}
\hline \multicolumn{3}{|c|}{ Condições Operacionais Teóricas } & $\begin{array}{c}\text { COV } \\
\left(\mathrm{kgDBO} \cdot \mathrm{m}^{-3} \cdot \mathrm{d}^{-1}\right)\end{array}$ & $\begin{array}{c}\text { DBO } \\
\left(\mathrm{mg} \cdot \mathrm{L}^{-1}\right)\end{array}$ & $\begin{array}{c}\text { TAS } \\
\left(\mathrm{m}^{3} \cdot \mathrm{m}^{-2} \cdot \mathrm{d}^{-1}\right)\end{array}$ & $\underset{(m)}{H}$ \\
\hline \multirow{8}{*}{ 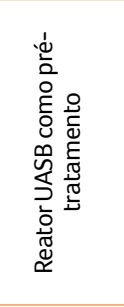 } & \multirow{2}{*}{ Cálculo 01} & A & 0,5 & 120 & 75 & 78 \\
\hline & & $\mathrm{B}$ & 0,5 & 120 & 50 & 12 \\
\hline & \multirow{2}{*}{ Cálculo 02} & A & 1,0 & 120 & 75 & 9 \\
\hline & & B & 1,0 & 120 & 100 & 12 \\
\hline & \multirow{2}{*}{ Cálculo 03} & A & 1,5 & 120 & 75 & 6 \\
\hline & & B & 1,5 & 120 & 150 & 12 \\
\hline & \multirow{2}{*}{ Cálculo 04} & A & 3,0 & 120 & 75 & 3 \\
\hline & & $\mathrm{B}$ & 3,0 & 120 & 300 & 12 \\
\hline \multirow{4}{*}{ 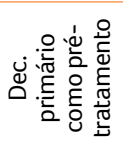 } & \multirow{2}{*}{ Cálculo 05} & A & 1,0 & 210 & 75 & 16 \\
\hline & & $\mathrm{B}$ & 1,0 & 210 & 57 & 12 \\
\hline & \multirow{2}{*}{ Cálculo 06} & A & 3,0 & 210 & 75 & 5 \\
\hline & & B & 3,0 & 210 & 171 & 12 \\
\hline
\end{tabular}

Legenda: A - fixação de TAS no limite estabelecido pela NBR 12.209/2011(75 m³. $\left.\mathrm{m}^{-2} \cdot \mathrm{d}^{-1}\right)$; B - fixação de H no limite estabelecido pela NBR $12.209 / 2011$ (12 m); COV - Carga Orgânica Volumétrica; DBO - Demanda Bioquímica de Oxigênio; TAS - Taxa de Aplicação Superficial; $\mathrm{H}$ - profundidade do meio suporte; valores tachados estão fora do limite da NBR 12.209/2011. 
Percebe-se que no Cálculo 01, para alcançar a mais elevada altura permitida para o meio suporte plástico, de $12 \mathrm{~m}$, a TAS é de $50 \mathrm{~m}^{3} \cdot \mathrm{m}^{-2} \cdot \mathrm{d}^{-1}$, pouco abaixo da faixa limite estabelecida pela Norma, de $75 \mathrm{~m}^{3} \cdot \mathrm{m}^{-2} \cdot \mathrm{d}^{-1}$. No Cálculo $02 \mathrm{com}$ elevação da COV, de 0,5 $\mathrm{kgDBO} \cdot \mathrm{m}^{-3} \cdot \mathrm{d}^{-1}$ para 1,0 $\mathrm{kgDBO} \cdot \mathrm{m}^{-3} \cdot \mathrm{d}^{-1}$, alcança-se a TAS máxima com altura de meio suporte de $9 \mathrm{~m}$. Nos Cálculos 03 e 04 a TAS máxima somente é alcançada com altura de meio suporte de $6 \mathrm{~m}$ e $3 \mathrm{~m}$, respectivamente. Assim, nessas duas últimas situações não se alcança a vantagem do dimensionamento de unidades mais esbeltas ocupando menores áreas em função de adoção de profundidades mais elevadas.

Chernicharo e Almeida (2011) estudaram diferentes configurações de sistemas do tipo UASBFBP com meio suporte sintético, considerando variações em cargas aplicadas (orgânicas e hidráulicas), além de diferentes alturas de meio suporte. Os autores consideraram unidade profunda aquela operada com altura do meio suporte de 4,2 $\mathrm{m}$ e, neste caso, as cargas impostas foram de TAS $=15 \mathrm{~m}^{3} \cdot \mathrm{m}^{-2} \cdot \mathrm{d}^{-1}$ e COV = 0,3 kgDBO $\cdot \mathrm{m}^{-3} \cdot \mathrm{d}^{-1}$. Assim, com esse valor imposto para TAS, a unidade operou com características de "Alta Carga", de acordo com os parâmetros determinados pela NBR 12.290/2011 e resumidos na Tabela 1 do presente texto. Entretanto, de acordo com a mesma fonte, em se tratando da carga orgânica, a unidade se aproximou mais de uma operação de "Baixa Carga". Para essas condições operacionais, as eficiências globais médias de remoção de SST, DQO e DBO foram de respectivamente $82 \%$, $89 \%$ e $90 \%$. Observa-se, sem dúvida, elevado desempenho global, entretanto não levando em consideração as principais vantagens associadas ao meio suporte plástico. Ao se adotar 4,2 m de profundidade de meio suporte, mesmo esta sendo relativamente superior ao limite máximo estabelecido para o meio em pedra ( $3 \mathrm{~m})$, ainda está bastante inferior aos $12 \mathrm{~m}$ estabelecidos pela NBR 12.209/2011 como limite para o meio plástico. Isso significa que não houve ganho sig- nificativo em relação à área em adotar um meio suporte sintético. Destaca-se, ainda, que os valores de TAS e COV adotados para este caso também se encontram bastante inferiores aos limites definidos para o filtro de alta taxa com meio suporte plástico, demonstrando mais uma vez o não alcance das principais vantagens associadas à adoção do meio suporte plástico. Conforme apresentado nos Cálculos 03 e 04 da Tabela 3 , com profundidade de meio suporte variando entre 3 e $6 \mathrm{~m}$, seria possível alcançar COV de 1,5 kgDBO.m $\mathrm{m}^{-3} \cdot \mathrm{d}^{-1}$ e $3,0 \mathrm{kgDBO} \cdot \mathrm{m}^{-3} \cdot \mathrm{d}^{-1}$, respectivamente e TAS de $75 \mathrm{~m}^{3} \cdot \mathrm{m}^{-2} \cdot \mathrm{d}^{-1}$ para ambos os casos. Com desempenho mantido, talvez fosse possível uma redução significativa de área ocupada.

Variações entre taxas aplicadas aos FBP e profundidades de meio suporte plástico em sistemas compostos por UASB-FBP também foram estudas por Rodriguez et al. (2011). Em 5 diferentes plantas com escala plena na Colômbia, as TAS estudadas foram de $30 \mathrm{~m}^{3} \cdot \mathrm{m}^{-2} \cdot \mathrm{d}^{-1}$, $49,8 \mathrm{~m}^{3} \cdot \mathrm{m}^{-2} \cdot \mathrm{d}^{-1}, 43,4 \mathrm{~m}^{3} \cdot \mathrm{m}^{-2} \cdot \mathrm{d}^{-1}, 53,1 \mathrm{~m}^{3} \cdot \mathrm{m}^{-2} \cdot \mathrm{d}^{-1}$ e $8,4 \mathrm{~m}^{3} \cdot \mathrm{m}^{-2} \cdot \mathrm{d}^{-1}$; e as profundidades de meio suporte foram de 4,0 m, 4,6 m, 4,16 m, 3,0 m e 4,3 m. Ressalta-se que exceto a última, todas as TAS aplicadas caracterizam unidades de "Alta Carga", bem como todas as profundidades acima de 3,0 m, de acordo com os parâmetros definidos pela NBR 12.209/2011 e resumidos na Tabela 1 do presente texto. Em relação às 5 plantas, os autores não apresentaram resultados individuais; entretanto as eficiências médias globais das 5 ETEs juntas, para SST, DQO e DBO, foram de respectivamente $81 \%, 81 \%$ e $80 \%$. Neste trabaIho, com adoção de taxas de aplicação superficial consideravelmente superiores àquela adotada por Chernicharo e Almeida (2011), observou-se desempenho global inferior. Ainda, em comparação com os resultados teóricos apresentados na Tabela 3, observa-se também uma relação entre taxa de aplicação superficial e profundidade do meio suporte. Entretanto, essa não é linear, talvez em função de diferentes cargas orgânicas 
volumétricas aplicadas, por se tratar de unidades operando em escala real. Com os cálculos teóricos apresentados na Tabela 3, em função da Eq. 3 , pode-se observar claramente a relação entre as variáveis de dimensionamento do FBP. Neste caso, com a manutenção da carga orgânica volumétrica, deveria se esperar uma relação diretamente proporcional entre taxa de aplicação superficial e profundidade do meio suporte.

No Cálculo 05, onde se tem uma maior carga orgânica afluente em função da unidade primária de tratamento ser menos eficiente para remoção de matéria orgânica, alcança-se a mais elevada altura de meio suporte, de $12 \mathrm{~m}$, com TAS de $57 \mathrm{~m}^{3} \cdot \mathrm{m}^{-2} \cdot \mathrm{d}^{-1}$. Porém isso acontece em função de uma COV reduzida. Quando se eleva a COV para o limite permitido pela Norma, de $3,0 \mathrm{kgDBO} \cdot \mathrm{m}^{-3} \cdot \mathrm{d}^{-1}$, conforme situação apresentada no cálculo 06 , a altura encontrada para a maior TAS possível é de $5 \mathrm{~m}$.

Para a configuração DP + FBP, não foi avaliada a $\operatorname{COV}=0,5 \mathrm{kgDBO} \cdot \mathrm{m}^{-3} \cdot \mathrm{d}^{-1}$ nem a $\mathrm{COV}=$ $1,5 \mathrm{kgDBO} \cdot \mathrm{m}^{-3} \cdot \mathrm{d}^{-1}$, estudadas na configuração UASB + FBP. Sabe-se que atualmente os projetos mais recentes que adotam FBP em seus fluxogramas raramente utilizam DP como unidade precedente, e sim o Reator UASB. Assim, para o fluxograma UASB + FBP, estudaram-se taxas intermediárias às limites, com o objetivo de observar o comportamento dos parâmetros de dimensionamento (COV, TAS e H) nos cálculos operacionais teóricos. De qualquer maneira, para a configuração UASB + FBP, adotando-se a menor $\operatorname{COV}\left(0,5 \mathrm{kgDBO} \cdot \mathrm{m}^{-3} \cdot \mathrm{d}^{-1}\right)$ e a maior TAS $\left(75 \mathrm{~m}^{3} \cdot \mathrm{m}^{-2} \cdot \mathrm{d}^{-1}\right)$, a profundidade já se apresentou bastante superior àquela máxima definida pela NBR 12209/2011 (12 m). Assim, de acordo com a Eq. 3, no caso da configuração DP + FBP, essa profundidade seria ainda maior para a mesma condição operacional teórica. $E$, ao se fixar a profundidade máxima de $12 \mathrm{~m}$ para a configuração $D P$ + FBP, a TAS seria bastante inferior àquela encontrada para a configuração UASB + FBP na mesma condição operacional teórica.

Percebe-se, assim, certa dificuldade em obter uma grande vantagem da aplicação do meio suporte plástico, que é a ocupação de áreas reduzidas em função da adoção de profundidade e TAS mais elevadas.

O sistema UASB-FBP operado por Fonseca et al. (2009) alcançou intervalos médios de eficiências de remoção de SST, DQ O e DBO de 96 a 98\%, 82 a $91 \%$ e 89 a $91 \%$, respectivamente. Esses intervalos são referentes às diferentes condições operacionais aplicadas à unidade, com TAS variando de 5 a $20 \mathrm{~m}^{3} \cdot \mathrm{m}^{-2} \cdot \mathrm{d}^{-1}$, em filtros preenchidos com meio suporte plástico e altura útil de $3 \mathrm{~m}$. Nesta pesquisa, os autores afirmam, por meio de testes estatísticos de variância do tipo Kruskal-Wallis, que os desempenhos não apresentaram diferenças significativas para as variações impostas ao sistema e, dessa forma, diferentes taxas impostas não afetaram o desempenho da unidade. Essa conclusão de Fonseca et al. (2009) corrobora a vantagem de aplicar taxas mais elevadas nos filtros preenchidos com meio suporte plástico com o objetivo de alcançar menores áreas ocupadas, garantindo bom desempenho. É importante destacar que de acordo com a Tabela 1, que resume parâmetros de dimensionamento definidos na NBR 12.209/2011, a unidade operada por Fonseca et al. (2009) esteve submetida, para meio suporte plástico, tanto à carga hidráulica considerada baixa $\left(<10 \mathrm{~m}^{3} \cdot \mathrm{m}^{-2} \cdot \mathrm{d}^{-1}\right)$ como à carga hidráulica considerada alta $\left(>10 \mathrm{~m}^{3} \cdot \mathrm{m}^{-2} \cdot \mathrm{d}^{-1}\right)$.

A possibilidade de aplicação de COV mais elevada também é considerada a principal vantagem do FBP com meio suporte plástico, porém ao elevá-la a valores próximos ao limite estabelecido pela Norma observa-se uma necessidade de redução na altura do meio suporte para se adequar a TAS ao limite superior indicado pela NBR 12.209/2011. 
Todos os cenários apresentados na Tabela 3 trabalham com TAS no limite superior estabelecido pela Norma. Cabe ressaltar o risco associado à operação da ETE com valores de TAS próximos ao limite permitido. Tal situação torna o FBP mais vulnerável a variações rotineiras de vazão e carga orgânica aplicada, podendo comprometer seu funcionamento e, consequentemente, a eficiência do tratamento. Nos cenários apresentados é possível então reduzir a TAS, reduzindo ainda mais a altura do meio suporte. Observa-se nos resultados apresentados por outros pesquisadores (CHERNICHARO E ALMEIDA, 2011; FONSECA et al., 2009; RODRIGUEZ et al., 2011) que em geral não são adotados valores de TAS, COV e profundidade próximos aos valores limites estabelecidos pela NBR 12.209/2011, nem mesmo para as unidades que adotam meio suporte plástico.

Conforme já mencionado, para meio suporte plástico, a NBR 12.209/2011 recomenda TAS variando entre $10 \mathrm{~m}^{3} \cdot \mathrm{m}^{-2} \cdot \mathrm{d}^{-1}$ e $75 \mathrm{~m}^{3} \cdot \mathrm{m}^{-2} \cdot \mathrm{d}^{-1}$, COV inferior a 3,0 $\mathrm{kgDBO} \cdot \mathrm{m}^{-3} \cdot \mathrm{d}^{-1}$ e profundidade do meio suporte inferior a $12 \mathrm{~m}$. Chernicharo e Almeida (2011) adotaram valores para TAS, COV e $\mathrm{H}$ bastante inferiores aos limites estabelecidos pela Norma. No caso da TAS, esse valor foi 5 vezes inferior; para COV, 10 vezes menor; e para $\mathrm{H}$, adotou-se valor de aproximadamente 3 vezes menor. Já Rodriguez et al. (2011) adotaram valores de TAS mais próximos do limite, entretanto não superiores a $50 \mathrm{~m}^{3} \cdot \mathrm{m}^{-2} \cdot \mathrm{d}^{-1}$, com profundidade de meio suporte em torno de $4 \mathrm{~m}$. Por fim, Fonseca et al. (2009) adotaram valores para TAS máxima e profundidade de meio suporte bastante inferiores aos limites indicados pela NBR 12.209/2001.

Alguns pesquisadores operaram sistemas de FBP com alta carga de acordo com todos os parâmetros definidos pela NBR 12.209/2011 (TAS, COV e H). Pontes e Chernicharo (2006) operaram um sistema de UASB seguido de FBP com preenchimento em polipropileno, TAS de $25,1 \mathrm{~m}^{3} \cdot \mathrm{m}^{-2} \cdot \mathrm{d}^{-1}$, COV de 2,0 kgDBO. $\mathrm{m}^{-3} \cdot \mathrm{d}^{-1}$ e $\mathrm{H}$ de $4,2 \mathrm{~m}$ alcan- çando eficiência global de remoção de SST, DQO e DBO de 88, 91 e 83\%, respectivamente. Já Santos et al. (2005) operaram FBP com profundidade de $3,0 \mathrm{~m}$, com cargas bastante elevadas, (TAS $<80 \mathrm{~m}^{3} \cdot \mathrm{m}^{-2} \cdot \mathrm{d}^{-1}$ e COV $<2,1 \mathrm{kgDBO} \cdot \mathrm{m}-$ $\left.{ }^{-3} \cdot \mathrm{d}^{-1}\right)$ e concluíram que a aplicação de TAS = $65 \mathrm{~m}^{3} \cdot \mathrm{m}^{-2} \cdot \mathrm{d}^{-1}$ e COV $=1,5 \mathrm{kgDBO} \cdot \mathrm{m}^{-3} \cdot \mathrm{d}^{-1}$ permite a obtenção de efluente com qualidade satisfatória, com concentrações de DBO e SST inferiores a $60 \mathrm{mg} / \mathrm{L}$. USEPA (1991) reportou um FBP operando com COV de $1,19 \mathrm{kgDBO} \cdot \mathrm{m}^{-3} \cdot \mathrm{d}^{-1}$, TAS de $42,7 \mathrm{~m}^{3} \cdot \mathrm{m}^{-2} \cdot \mathrm{d}^{-1}$ e altura de 9,8 $\mathrm{m}$ após decantador primário com DBO afluente de $122 \mathrm{mg} / \mathrm{L}$, chegando a uma remoção de 59 \% de DBO.

Assim, é possível observar que a aplicação de cargas hidráulicas e orgânicas elevadas (dentro dos intervalos estabelecidos pela NBR 12.209/2011) sugerem bons desempenhos para a remoção de matéria orgânica para ambas as variáveis (COV e TAS), com ocupação de áreas reduzidas.

Por fim, ao se fixar a COV no valor máximo permitido pela Norma $\left(3,0 \mathrm{kgDBO} \cdot \mathrm{m}^{-3} \cdot \mathrm{d}^{-1}\right)$, com a utilização do reator UASB como tratamento primário avançado, a profundidade não passa de $3 \mathrm{~m}$. Neste caso, questiona-se inclusive a utilização do meio suporte plástico em detrimento do meio em pedra, que tem custo mais reduzido e ocuparia área similar. Não obstante, deve-se atentar para aspectos construtivos referentes aos dois materiais de preenchimento. 0 meio suporte em pedra demanda estrutura de sustentação mais robusta com um fundo falso que deve suportar todo o peso do material de preenchimento e, além disso, as interveniências decorrentes da manutenção e reparo de tais estruturas podem ser mais onerosas. É importante, ainda, discutir a área superficial específica do meio suporte plástico, que é consideravelmente superior à do meio suporte em pedra (JORDÃO E PESSÔA, 2017). Por esse motivo, os filtros preenchidos com meio suporte plástico são capazes de manter uma maior densidade de microrganismos por unidade de 
área, garantindo maior taxa de degradação de matéria orgânica.

Destaca-se ainda o trabalho desenvolvido por Borba et al. (2017), que teve o objetivo de comparar dois métodos de dimensionamento de filtros biológicos percoladores com meio suporte plástico precedidos por reatores UASB, a saber: i) o método adotado no Brasil já amplamente discutido neste artigo; e ii) o método DWA 2016, desenvolvido pela Deutsch Vereinigungfür Wasserwirtschaft, Abwasserund Abfall (Associação Alemã para Gerenciamento de Água, Águas Residuárias e Resíduos). Este último é um método otimizado, não apenas para a realidade alemã, mas sim para diferentes regiões climáticas, que consiste na adequação de equações e no cálculo da degradação da matéria orgânica e de nitrogênio amoniacal, por segmento de altura do meio suporte. Segundo os autores, o método torna o cálculo mais preciso para cada região do planeta. Para o exemplo de dimensionamento adotado no estudo, foi possível observar uma redução no volume de $11 \%$ em relação aos dois métodos estudados. Dessa forma, os autores afirmam que essa maior precisão se dá principalmente em função dos diferentes estágios de degradação da matéria orgânica, por populações microbianas distintas, que se estabelecem ao longo da profundidade da unidade.

\section{CONCLUSÕES}

O trabalho apresentou uma discussão teórica sobre os parâmetros envolvidos no dimensionamento do FBP. Entretanto, há que se ressaltar que não se esgotaram as possibilidades de combinações entre os valores adotados para as variáveis elencadas no presente estudo (COV, TAS e H).

Considerando o FBP precedido pelo Reator UASB, configuração cada vez mais utilizada no Brasil, pôde-se concluir que o limite de altura permitido pela Norma, de $12 \mathrm{~m}$, torna-se inviável pe- los elevados valores de TAS obtidos pela imposição do valor de $\mathrm{COV}$, que deve ser superior a $1,0 \mathrm{kgDBO} \cdot \mathrm{m}^{-3} \cdot \mathrm{d}^{-1}$. Para esse valor, a máxima profundidade atingível foi de apenas $9,0 \mathrm{~m}$. Somente foi possível a obtenção de uma altura igual a $12 \mathrm{~m}$ com valor de TAS abaixo do limite da Norma, utilizando um valor muito baixo para $\operatorname{COV}\left(0,5 \mathrm{kgDBO} \cdot \mathrm{m}^{-3} \cdot \mathrm{d}^{-1}\right)$. Ainda, adotando-se os limites superiores de TAS e COV normatizados, a profundidade máxima alcançada foi de $3 \mathrm{~m}$, que é o limite superior para o meio suporte em pedra.

Contudo, percebe-se que a adoção de profundidades elevadas, que consiste em uma das vantagens do FBP com meio suporte em plástico, quando comparada ao preenchimento do meio suporte de pedra, é inviabilizada. Tal fato decorre da dificuldade do atendimento concomitante dos parâmetros previstos da NBR 12.209/2011. Não obstante, deve-se atentar às outras vantagens do FBP, como a possibilidade de estruturas de sustentação menos robustas e facilidade de manutenção.

Ressalta-se ainda que a utilização de valores demasiadamente elevados de COV pode levar a problemas operacionais. Dessa forma, entendese que os FBP de alta carga devem ser dimensionados com meio suporte plástico prevendo alturas máximas entre 5,0 e 6,0 m, levando a taxas de dimensionamento mais seguras.

Sendo assim, conclui-se que a simples adoção de uma profundidade com vistas à elevação ou redução do volume de meio suporte para adequação de área deve ser avaliada com cautela, uma vez que o não atendimento dos valores máximos/ mínimos de TAS e COV preconizados na norma comprometem a eficiência do tratamento.

\section{CONTRIBUIÇÃO DOS AUTORES}

Todos os autores contribuíram de forma igualitária. 


\section{REFERÊNCIAS}

ABNT. Norma Brasileira NBR 12.209 - Elaboração de projetos hidráulico-sanitários de estações de tratamento de esgotos sanitários. Associação Brasileira de Normas Técnicas. Rio de Janeiro, RJ, 2011.53p.

ABNT. Norma Brasileira NBR 12.209 - Projeto de estações de tratamento de esgoto sanitário. Associação Brasileira de Normas Técnicas. Rio de Janeiro, RJ, 1992.12p.

ALMEIDA, P. G. S. Efeito de diferentes tipos de meio suporte no desempenho de filtros biológicos percoladores aplicados ao pós-tratamento de efluentes de reatores UASB, com ênfase na nitrificação. Dissertação (Mestrado em Saneamento, Meio Ambiente e Recursos Hídricos). Universidade Federal de Minas Gerais. Belo Horizonte, 2007.

ANA - AGÊNCIA NACIONAL DE ÁGUAS. Atlas Esgotos: Despoluição de Bacias Hidrográficas. Brasília, DF, 2017. 88p.

BORBA, A. L. B.; NEUFFER, K. J.; ROSS, B. Z. L. O Método Internacional DWA - 2016 para Dimensionamento de Filtros Percoladores e as Diferenças com os Métodos Praticados no Brasil: Comparação entre Resultados e a Influência do Meio Suporte. In: Congresso ABES/FENASAN 2017. São Paulo, outubro de 2017. Anais...

BORGES, E. A. M. A. Dimensionamento e Parametrização dos Serviços para a Implantação de Estações de Tratamento de Esgotos para Municípios de Pequeno Porte. Dissertação (Mestrado Profissional em Engenharia Ambiental). Universidade do Estado do Rio de Janeiro - PEAMB/UERJ. Rio de Janeiro, 2017.

CHERNICHARO, C. A. L.; FLORÊNCIO, L.; BASTOS, R. K. X.; PIVELI, R. P. SPERLING, M.; MONTEGGIA, L. O. Tratamento de Esgotos e Produção de Efluentes Adequados a Diversas Modalidades de Água de Reuso. In: Tratamento e utilização de esgotos sanitários. Rio de Janeiro, ABES 2006. 427p.

CHERNICHARO, C. A. L.; ALMEIDA, P. G. S. Feasibility of UASB/ trickling filter systems without final clarifiers for the treatment of domestic wastewater in small communities in Brazil. Water Science and Technology, v. 64, p. 1347-1354, 2011. https://doi. org/10.2166/wst.2011.389

COMPANHIA DE SANEAMENTO DE MINAS GERAIS (COPASA). ETE Onça: Marco do Saneamento Nacional. Belo Horizonte, 2006. Link acessado em junho de 2018: http://www.copasa.com.br/ wps/portal/internet/imprensa/noticias/releases/2006/junho/ ete-onca-20060607-ie763/!ut/p/a1/vZFNU8IwEIZ_C4ceM7v0K VYUfkoilMH016cmISSTkILGxz996aMHJHxYnLI7s47u3nehQ̨IYFIZ_6JJb3RheD3kRv60wS-dZhsvNdvGlabbdzDaLu-3TmsIrFFAIY1u7h7zXVhHRtLzn7jFWnWTTe6hd1BIIPWxVt901qxzaTpmee2gaq4XmTtWpWvFeuchHjD2sTmbfeKhcy8YIToaqu5RoReNgGNvyUknV69KcM6El5CKSIOQpfklhNQ hJGShluKCVBGGPAZeC_ y9BR5Y4Kr5wUb0EvbwncAL9bT9fl8Em7J9rsGmAXVmAXVmADFbAzK7BrrK6dro7HInVWD7Z-WmD_6fV5yY74GRFTcTjDZTLHBNOEjifzKOQZROP4R_GJp7jynVz17oPDyxyW2hONS7VZRXd5_BVhFbTkafQMalojG/dl5/d5/L2dBISEvZOFBIS9nQSEh/
DUDA, R.M.; OLIVEIRA, R.A. Tratamento de Águas Residuárias de Suinocultura em Reator UASB e Filtro Anaeróbio em Série Seguidos de Filtro Biológico Percolador. Revista Engenharia Sanitária e Ambiental, v.16, n.1, jan/mar de 2011. http://dx.doi.org/10.1590/ S1413-41522011000100013

FONSECA, M. F. Remoção de Nitrogênio Amoniacal em Filtro Biológico Percolador Pós-Tratando Efluente de Reator UASB. Dissertação (Mestrado em Engenharia Civil) -COPPE da Universidade Federal do Rio de Janeiro, Rio de Janeiro, 2009.

HENRICH, C. D.; MARGGRAFF, M. Energy-efficient Wastewater Reuse - The renaissance of Trickling Filter Technology.12 p. 2013. Disponível em: http://waterandcarbon.com.au/wp-content/uploads/2015/11/Henrich-2013-Paper-TF-IWA-Water-reuse-conference-Namibia-V10.pdf. Acessado em: 16/10/2020.

JORDÃO, E.P. \& PESSÔA, C.A. Tratamento de Esgotos Domésticos. 8.ed. Rio de Janeiro: ABES, 2017.1050p.

MORAES, O. J. S.; SOUZA, J. R.; SILVA, L. R.; AZEVEDO, S. G.; CHERNICHARO, C. A. L.; LOBATO, L. C. S.; SILVA, R. V. Long term performance of the largest Brazilian combined anaerobic/aerobic treatment plant ( $P E=1$ million inhabitants). In: Proc. X Daal - Latin American Workshop and Symposium on Anaerobic Digestion. Ouro Preto, 2011. Anais...

PONTES, P.P.; CHERNICHARO, C.A.L. Avaliação de desempenho de um sistema reator uasb - filtro biológico percolador operando com e sem decantador secundário. In: XXX Congreso Interamericano de Ingeniería Sanitaria y Ambiental. Punta del Este - Uruguay. 2006. Anais...

RIBEIRO, T. B.; ALMEIDA, P. G. S.; VOLCKE, E. I. P.; CHERNICHARO, C. A. L. Trickling filters following anaerobic sewage treatment: state of the art and perspectives. Water Res. Technol., v. 4p. 17211738, 2018. https://doi.org/10.1039/C8EW90041H

RODRIGUEZ, J. A.; TORRES, P.; DUQUU, A. Evaluation of UASB Reactor Followed by Trickling Filter for Domestic Wasterwater Treatment in Valle del Cauca, Colombia. In: Proc. X DAAL - Latin American Workshop and Symposium on Anaerobic Digestion. Ouro Preto (Minas Gerais), 2011. Anais...

SANTOS, A.S. Avaliação do Desempenho de um Filtro Biológico Percolador em Diferentes Meio Suporte Plásticos. 2005. 81 p. Dissertação (Mestrado em Ciência em Engenharia Civil). COPPE/ UFRJ, Rio de Janeiro, 2005.

SANTOS, A.S.; VOLSCHAN JR., I.; JORDÃO, E.P.; AZEVEDO, J.P.S. Desempenho do filtro biológico percolador segundo dois diferentes meios suporte plástico. In: $23^{\circ}$ Congresso Brasileiro de Engenharia Sanitária e Ambiental. Campo Grande/MS, 2005. Anais...

SPERLING, M. Introdução à qualidade das águas e ao tratamento de esgotos. $3^{\mathrm{a}}$ ed. Belo Horizonte: Departamento de Engenharia Sanitária e Ambiental, 2005. 452 p. (Princípios do Tratamento Biológico de Águas Residuárias). 
SPERLING, M. Princípios Básicos do tratamento de Esgoto. $2^{\mathrm{a}}$ ed. Belo Horizonte: Departamento de Engenharia Sanitária e Ambiental, 2016. 211 p. (Princípios do Tratamento Biológico de Águas Residuárias).

UNIVERSIDADE DE SÃO PAULO (USP). Software Auxilia Municípios na Escolha da Estação de Tratamento de Esgotos. In: Jornal da USP, 24 de maio de 2018. Editoriais: Ciência e Tecnologia. São Paulo, 2018.

VIEIRA, P. C.; von SPERLING, M. Open trickling filter: an innovative, cheap and simple form of post-treatment of sanitary effluents from anaerobic reactors in small communities. Journal of Water,
Sanitation and Hygiene for Development, v. 2, p. 59-67, 2012. https://doi.org/10.2166/washdev.2012.072

VIEIRA, P.C. Estudo do Comportamento de um Filtro Biológico Percolador com Laterais Abertas Aplicado ao Pós-Tratamento de Efluentes de Reator UASB. Tese (Doutorado em Saneamento, Meio Ambiente e Recursos Hídricos). Universidade Federal de Minas Gerais. Belo Horizonte, 2013.

USEPA, United States Environmental Protection Agency. Assessment of single-stage trickling filter nitrification. Washington D.C., 105 p, 1991. 